\title{
Density distribution in medium-sized icy satellites of giant planets
}

\author{
Leszek Czechowski ${ }^{1}$ and Jacek Leliwa-Kopystynski ${ }^{1,2}$ \\ ${ }^{1}$ University of Warsaw, Institute of Geophysics, ul. Pasteura 7, 02-093 Warszawa, Poland \\ ${ }^{2}$ Space Research Centre of the Polish Academy of Sciences, ul. Bartycka 18A, 00-716 Warszawa, Poland \\ (Received September 4, 2006; Revised May 15, 2007; Accepted July 8, 2007; Online published September 28, 2007)
}

\begin{abstract}
We discuss processes that determine the distribution of density in the medium-sized icy satellites (MIS). Gravitational differentiation, porosity and phase transitions lead to a spherical distribution, while thermal convection, large impacts and tidal deformation can result in a non-spherical distribution. According to our previous research, convective patterns in MIS can consist of one or two convective cells for radiogenic and tidal heating. The shift of the center of mass $\Delta r_{\mathrm{CM}}$ and the ratio of moments of inertia $I_{Z} / I_{X Y}$ are calculated using a numerical model of convection. A new dimensionless number $C$ is introduced to describe the deformation of the surface. We found that $\Delta r_{\mathrm{CM}}$ can reach $\sim 0.5 \%$ of the satellite radius for the one-cell pattern. With the two-cell pattern the moment of inertia, $I_{Z}$, can be reduced by $\sim 0.4 \%$. The impact cratering could be one cause of significant changes in $\Delta r_{\mathrm{CM}}$ and $I_{Z} / I_{X Y}$ but only for the smallest of the MIS. Tidal deformation could result in the enhancement of mass redistribution caused by other mechanisms.
\end{abstract}

Key words: Icy satellites, density distribution, convection, tidal heating, moments of inertia.

\section{Introduction}

Icy satellites are naturally divided into three size classes. Large satellites have radii exceeding approximately $1200 \mathrm{~km}$, and small satellites have irregular shapes. In the present paper we consider medium sized icy satellites (MIS). The lower limit of radius of the MIS corresponds to the smallest spherical bodies (radius of Mimas is $199 \mathrm{~km}$ ). Titania $(789 \mathrm{~km})$ is the largest MIS.

The shape of a celestial body and its internal density distribution depend on several factors. If the mass of an icy body exceeds $10^{19}-10^{20} \mathrm{~kg}$, then gravity, pressure and temperature inside the body are high enough to make its interior behave like a very viscous fluid, leading to the spherical shape of the body. This fluid-like behaviour is exhibited only by the deep material. The upper layers could behave in a different way. In general, the distribution of density could be determined by several processes, including gravitational differentiation (sinking of denser material), phase changes and closing of the voids (the pores that certainly exist at least in the near-surface layers). Moreover, the nonspherical component of density distribution could be a result of thermal convection, impact cratering and tidal deformation.

Density distribution in any celestial body is one of its basic characteristics. The density distribution in the MIS has been the subject of many papers (e.g. Kossacki and LeliwaKopystynski, 1993) but most of these consider processes that lead to a spherically symmetric distribution. Nonspherical distributions are usually discussed in connection with the tidal bulge (e.g. Zharkov et al., 1985). For studies

Copyright (c) The Society of Geomagnetism and Earth, Planetary and Space Sciences (SGEPSS); The Seismological Society of Japan; The Volcanological Society of Japan; The Geodetic Society of Japan; The Japanese Society for Planetary Sciences; TERRAPUB. of convection in the icy satellites see, for example, McKinnon (1998) and Sotin et al. (1998). In the present paper we investigate mainly the role of the solid-state convectionthe most complicated and rarely considered process. The role of heat transfer by conduction and convection in the MIS has been a subject of many papers. The existence of convection was often neglected because of the small size of the MIS. Ellsworth and Schubert (1983), however, found that small satellites could be conductive (Mimas), and since the publication of their paper knowledge on the MIS has increased enormously. The internal activity in Enceladus (confirmed by Cassini mission) indicates a considerable role of convection. Let us also stress that solid-state convection in the MIS is much more probable than convection in a liquid interior. Of course, we do not deny the possibility of the presence of some limited volume of cryo-magma, but its role in global convection seems to be negligible (like the role of the silicate magmas in the Earth's mantle). In this paper, we use a numerical model of convection and compare the role of convection with the role of the other processes.

\section{Processes that Lead to Spherically Symmetric Density Distributions}

There are several processes responsible for the evolution of the icy satellites. Their roles depend on the satellite mass, ice-to-rock mass ratio, intensity of tidal interaction with the primary body, among others. The history of formation of a given satellite is crucial for its evolution as it provides the initial conditions (the end of the accretion is a starting point for satellite evolution). It is reasonable to assume that the satellites were formed from the uniform icy-mineral matter of pre-satellite circum-planetary nebulae. Formation of the MIS was much faster than their post-accretion evolution. Therefore, we can neglect the evolutionary processes during the accretion. Accreting material settling on a growing 
satellite surface forms concentric, homogenous, porous layers with increasing temperature as the square of the central distance (Ellsworth and Schubert, 1983). It is plausible that inside the largest MIS the temperature exceeds the water-ice melting point. If so, the porosity disappears and a differentiation develops due to mineral grains sinking into the molten medium. The porosity could also disappear in solid bodies as a result of the fluid-like behaviour of the interior (solidstate creeping). In such a case, the differentiation is dramatically reduced. Those processes are continuously disturbed by the heat transfer and by the radiogenic heat production. The near-surface layer could remain porous. In the smallest MIS, the thickness of the porous layer could be as large as 0.1 of its radius. Mimas with a porosity $\sim 0.5$ in the uppermost layer of thickness, $\sim 0.15$ of the radius, is an example (Leliwa-Kopystynski and Kossacki, 2000). In the calculation of convection we assume homogeneity of the satellites. Therefore, our results do not relate to the largest of the MIS (Rhea, Iapetus, Titania, and Oberon).

The process of phase transitions of water-ice in the MIS may develop on the surface or in the deep interior. On the one hand, if accreting material contains amorphous ice then it transforms to the hexagonal form (ice I) at the time of its settling on the surface of the accreting satellite. The latent energy of this phase transition can be included in a boundary condition on the satellite surface. On the other hand, if the pressure in the satellite's interior reaches $\sim 200 \mathrm{MPa}$, then ice I could transform into a higher-pressure polymorph. This pressure could be found only in the deep interior of the largest MIS (e.g. Leliwa-Kopystynski et al., 1994). In any case, due to the $p, T$ conditions, most waterice in the interior of the MIS remains in the form of ice I. Therefore, the phase transitions play only minor role in the evolution of the MIS. The situation could be more complicated if other types of ice are present. Note that the possible presence of ammonia in Enceladus (widely considered at present) strongly influences its internal evolution. An admixture of ammonia is important when considering convection as it can significantly reduce the viscosity of the medium. However, the water-ammonia system in the $p, T$ conditions corresponding to most of the MIS interiors is not undergoing phase transitions (Leliwa-Kopystynski et al., 2002).

Let us consider the interaction of the gravitational differentiation and the solid-state convection (e.g. Czechowski, 1992, 1993). The main process of differentiation (if any) in MIS is a sinking of silicate rocks, leading to the formation of a rocky core. The velocity of sinking can be estimated using the Stokes formula (e.g. Stacey, 1992, p. 271, eq. 5.97) that states: $F=6 \pi \eta r v_{\text {sinking, }}$, where $F$ is the force acting on a sphere of radius $r$ moving with the velocity $v$ in a liquid with the viscosity $\eta$. In our case $F$ is a buoyancy force so: $F=(4 / 3) \pi r^{3} g \Delta \rho$ where $\Delta \rho$ is the density difference and $g$ is the gravity. Comparing both formulas we find that the velocity of sinking is $v_{\text {sinking }}=2 r^{2} g \Delta \rho /(9 \eta)$. The characteristic size $r$ of rocky grains or boulders in MIS is unknown. Assuming that silicates form boulders with size $r<10^{2} \mathrm{~m}$, we find that $v_{\text {sinking }}$ is less than approximately $2 \times 10^{-13} \mathrm{~m} \mathrm{~s}^{-1} \approx 6 \times 10^{-3} \mathrm{~mm} \mathrm{y}^{-1}$ (for $\eta=10^{18} \mathrm{~kg} \mathrm{~m}^{-1} \mathrm{~s}^{-1}, \Delta \rho=10^{3} \mathrm{~kg} \mathrm{~m}^{-3}, g=0.1 \mathrm{~m}$ $\left.\mathrm{s}^{-2}\right)$. Such $v_{\text {sinking }}$ is negligible compared to the velocity of slow convection, i.e. $1 \mathrm{~mm} \mathrm{y}^{-1}$. The low value of $v_{\text {sinking }}$ means that differentiation is negligible for the solid interiors of MIS.

\section{Tidal Deformation}

All of the MIS rotate synchronously, i.e. their orbital periods are equal to their spin periods. The gravity of the planet and the centrifugal force it exerts result in a deformation of the satellite from a sphere to a tri-axial ellipsoid (e.g. Zharkov et al., 1985; Eluszkiewicz and LeliwaKopystynski, 1989). The magnitude of the distortion is approximately (5-10)\% for Mimas (Zharkov et al., 1985; Dermott and Thomas, 1988) and about 3\% for Enceladus (Zharkov et al., 1985) and is probably less than $2 \%$ for larger MIS. These are significant values compared to the values given by other causes of non-spherical shape. However, there is an important difference: tidal deformation always acts in the same direction, i.e. it elongates a satellite along the satellite-planet line. However, the position of this line with respect to the internal as well the surface structures of the satellite are determined by already existing non-spherical structures. Therefore, the tidal force could be treated as an amplifier of the other existing causes of nonspherical density distribution.

\section{Numerical Model of Convection}

We use a numerical model to investigate the effect of convection on density distribution. The model is based on the following system of equations: the Navier-Stokes equation, the equation of thermal conductivity, the equation of continuity and the equation of state. The OberbeckBoussinesq approximation of the equations is used here (see e.g. Czechowski, 1993; Schubert et al., 2001):

$$
\begin{aligned}
& \rho_{0} \frac{D \boldsymbol{v}}{D t}=-\nabla p+\nabla \cdot(2 \eta \boldsymbol{D})+\rho \boldsymbol{g}, \\
& \frac{\partial T}{\partial t}+\boldsymbol{v} \cdot \nabla T=\kappa \nabla^{2} T+Q / c, \\
& \nabla \cdot \boldsymbol{v}=0, \\
& \rho=\rho_{0}(1-\alpha T),
\end{aligned}
$$

where $t, \boldsymbol{v}, \boldsymbol{D}, p, T, \rho, \rho_{0}, \alpha, \eta, \boldsymbol{g}, c, Q, \kappa$ denote the time, velocity, tensor of deformation rate, pressure, temperature, density, reference density, coefficient of thermal expansion, viscosity, gravity, specific heat, efficiency of heat sources per unit mass and coefficient of thermal diffusion, respectively. The tensor $\boldsymbol{D}$ can be expressed by the velocity and its derivatives. In the following text we use $q=Q / c\left[\mathrm{~K} \mathrm{~s}^{-1}\right]$ instead of $Q$. The gravity vector $\boldsymbol{g}$ is a function of distance $r$ from the centre of the body. Because a non-differentiated satellite is considered here, the gravity is proportional to $r$ and is expressed by the formula $\boldsymbol{g}=-g_{0}(r / d) \boldsymbol{i}_{r}$, where $g_{0}$, $d, \boldsymbol{i}_{r}$, are the gravitational acceleration at the surface, the radius of the body and the unit radial vector (directed outward of the center), respectively. Note that changes in density resulting from convection are negligible compared to $\rho_{0}$ and, therefore, can be neglected in the calculation of $\boldsymbol{g}$; this is a typical assumption in convective models (the OberbeckBoussinesq approximation). 
Let us define the following natural units of time, length, velocity, stream function, temperature and pressure ( $\eta_{0}$ is a reference viscosity, $q_{\text {av }}$ is globally averaged $q$ ):

$$
\begin{aligned}
& \tau=d^{2} / \kappa, \quad \lambda=d, \quad \omega=\kappa / d, \\
& \Psi=\kappa, \quad \Theta=q_{\mathrm{av}} d^{2} / \kappa, \quad \sigma=\eta_{0} \kappa d^{-2} .
\end{aligned}
$$

The system of Eqs. (1)-(4) can be transformed into dimensionless form by means of the following substitutions (primed variables are dimensionless, i.e. given in the natural units)

$$
\begin{aligned}
& t=t^{\prime} \tau, \quad r=r^{\prime} \lambda, \quad v=v^{\prime} \omega, \\
& T=T^{\prime} \Theta+80 \mathrm{~K}, \quad p=p^{\prime} \sigma .
\end{aligned}
$$

Here, the temperature $80 \mathrm{~K}$ is the reference temperature corresponding approximately to the surface temperature. Transformation (6), the introduction of non-hydrostatic pressure $p_{\mathrm{nh}}$, and the substitution of the equation of state into the Navier-Stokes equation reduce the number of the system parameters to two dimensionless numbers: the Rayleigh number $R a$ and Prandtl number $P r$. For solidstate convection, $P r$ is very large and can be assumed to be infinite. This allows the non-inertial terms in the NavierStokes equation to be neglected. Finally we obtain the following dimensionless system:

$$
\begin{aligned}
& 0=-\nabla p_{\mathrm{nh}}{ }^{\prime}+\nabla \cdot\left(2 \eta^{\prime} \boldsymbol{D}^{\prime}\right)+R a T^{\prime} r^{\prime} \boldsymbol{i}_{r}, \\
& \frac{\partial T^{\prime}}{\partial t^{\prime}}+\left(\boldsymbol{v}^{\prime} \cdot \nabla T^{\prime}\right)=\nabla^{2} T^{\prime}+q^{\prime}, \\
& \nabla \cdot \boldsymbol{v}^{\prime}=0,
\end{aligned}
$$

where $q^{\prime}=q / q_{\mathrm{av}}, \eta^{\prime}=\eta / \eta_{0}$. The Rayleigh number $R a$ is

$$
R a=\rho_{0} g_{0} \alpha q_{\text {av }} d^{5}\left(\eta_{0} \kappa^{2}\right)^{-1} .
$$

If steady-state convection is assumed, then the time derivative in the equation of heat transfer (8) is equal to zero. For the case of constant viscosity, $\eta^{\prime} \equiv 1$.

To solve the system L. Czechowski developed a numerical code using the finite difference method. The spherical system of coordinates $\left(r^{\prime}, \vartheta, \varphi\right)$ with the origin at the centre of mass of the satellite is used for discretization. Equations (7)-(9) are solved in a sphere of radius $r^{\prime}=1$. A special numerical scheme is applied for $\vartheta \approx 0$ and $\vartheta \approx \pi$ (i.e. close to the $z$-axis) to reduce the numerical effects typical for non-uniform grids. Two different boundary conditions (BC) are considered at the upper boundary, i.e. at $r^{\prime}=1$ : (1) free $\mathrm{BC}$, i.e. tangential stresses are zero at the upper boundary, and (2) zero-velocity BC, i.e. $v=0$ at the upper boundary. Moreover, an isothermal boundary $T^{\prime}=0$ is assumed at $r^{\prime}=1$. For more details of the model, see Czechowski and Leliwa-Kopystynski (2005).

\section{General Properties of Convection}

In the present work we discuss constant viscosity models. There are a few reasons for that:

(1) The problem of mass redistribution resulting from convection has not been discussed to date. We therefore feel obliged to present the simplest cases that provide a better insight into the basic properties of this phenomenon.

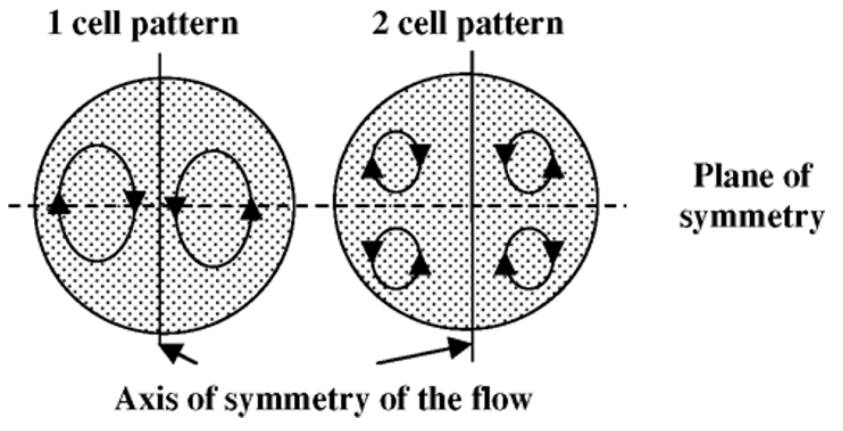

Fig. 1. The two basic patterns of convection typical of low and moderate Rayleigh number: the one-cell pattern and the two cell-pattern. The regions close to the axis of symmetry are referred to as 'polar regions'. The convective patterns have a plane of symmetry ('equatorial region') perpendicular to the axis of symmetry. Note that the symmetry of the convective pattern is not ideal, especially for non-uniform tidal heating.

(2) The constant-viscosity models are described by the lowest possible number of parameters without any loss of physical meaning.

(3) The scaling is possible even for time-dependent models.

The main difference between constant viscosity and temperature-dependent viscosity can be summarized as follow: if the viscosity contrast is high, then the stagnant lid is formed on the surface. The region below the lid is almost isoviscous (e.g. Schubert et al., 2001). Consequently, the constant viscosity model can be applied, but (in that case) the Rayleigh number $R a$ should be calculated for lower value of $d$ (compare Eq. (10)) since there is no convection in the stagnant lid.

In our model, the heat generation rate is a sum of two terms representing two different physical phenomena responsible for the heating: the decay of radioactive elements $q_{r}$ and the heat generation resulted from tidal deformations $q_{t}$ :

$$
q(r, \theta, \varphi)=q_{r}+q_{t}(r, \theta, \varphi) .
$$

The method of calculating tidal heating (based on Peale et al. (1979) and Poirier et al. (1983)) was presented by Czechowski and Leliwa-Kopystynski (2003, 2005). In the following we use the globally averaged dimensionless ratio $L=q_{t} / q(0 \leq L \leq 1)$.

According to our previous papers the patterns of convection driven by the tidal heating and convection driven by the radiogenic heating are generally similar for the range of $R a$ considered here (from $\sim 10^{4}$ to $\sim 3 \times 10^{5}$ ). Two patterns are observed: the one-cell pattern and two-cell pattern; see Fig. 1. The one-cell pattern has one region of downward motion and one region of upward motion. The two-cell pattern has two separate regions of downward motion and one common region of upward motion. The general similarity of the flow for such different modes of heating is rather unexpected. This similarity justifies limiting our calculations to uniform heating. The main differences between the two modes of heating are:

(1) There is no orientation for the homogenous radiogenic heating while tidal heating gives the pattern strongly 
(a)

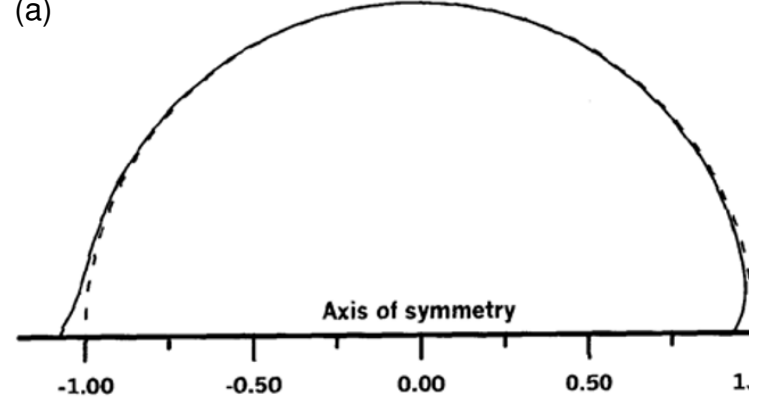

(b)

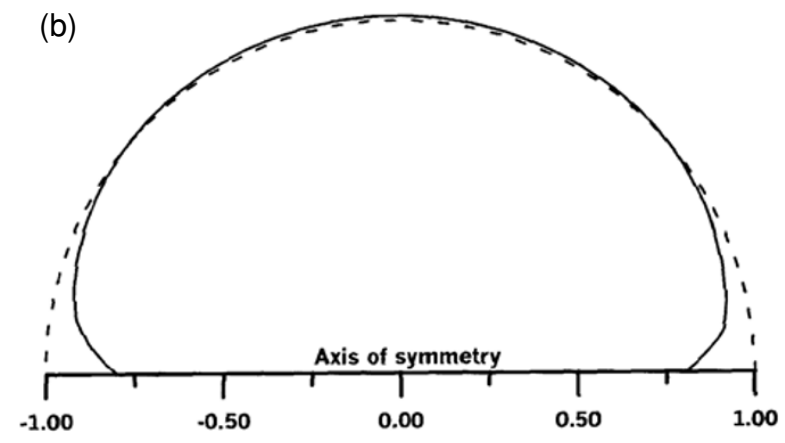

Fig. 2. The deformation of the surface of a satellite (magnified 100 times) for the free BC: (a) One-cell pattern for $R a=30,000$, (b) two-cell pattern for $R a=210,000$. The compensation number is $C=4 \times 10^{-6}$.

oriented in respect to the planet-satellite line.

(2) The rate of radiogenic heating is strictly limited by the content of radioactive elements and is decreasing over time. The tidal heating has no such limitations: it can be very intensive and can operate at any time.

Note that these differences are not crucial for the mass distribution problem because the patterns as well as the velocities in both models of convection for the same values of $R a$ are similar.

\section{Surface Deformation and the Compensation Number $C$}

The total changes in density distribution resulting from convection are the sum of two effects: an internal contribution resulting from the distribution of temperature inside the satellite and that from the surface contribution caused by the deformation of the satellite's surface. The surface deformation is calculated from the condition that the pressure of elevated mass $\Delta p=\rho_{\mathrm{s}} g_{0} \Delta h$ is compensated by the normal component of the stress tensor $\boldsymbol{T}$, i.e.

$$
\rho_{\mathrm{s}} g_{0} \Delta h=\boldsymbol{i}_{r} \cdot \boldsymbol{T} \cdot \boldsymbol{i}_{r},
$$

where $\Delta h$ is the vertical deformation of the surface and $\rho_{\mathrm{s}}, g_{0}$ are near-surface density (at the bottom of the regolith layer) and the surface gravity acceleration, respectively. The dimensionless deformation is given by

$$
\Delta h^{\prime}=C T_{r r^{\prime}}{ }^{\prime}
$$

where dimensionless parameter $C$ is referred as the 'compensation' number. It is defined as follows

$$
C=\eta_{\mathrm{s}} \kappa \rho_{\mathrm{s}}^{-1} g_{0}^{-1} d^{-3} .
$$

The number $C$ describes the 'sensitivity' of the surface with respect to the stresses resulting from convection. The magnitude of the surface deformation depends on both $R a$ and $C$. The product $R a C$ can be used as a rough measure of the dimensionless deformation.

Figure 2 presents typical deformations of the surface. The calculation of convection at the satellite surface is performed assuming a non-deformed sphere. The surface's deformation is calculated using the stress tensor obtained from the model of convection. Coupling the convection and the surface deformation gives higher order effects since the deformation is negligible compared to the size of the sphere. Note that depressions appear above the downward convective currents while elevations are above the upward currents. In the case of the one-cell pattern, the regions of depression and elevation are almost symmetric. In the case of the two-cell pattern, the depressions are concentrated close to the 'polar' regions while one elevation forms a wide belt around the 'equatorial' region.

Let us discuss the possible range of $C$. For Dione, $\rho_{\mathrm{s}}=$ $1490 \mathrm{~kg} \mathrm{~m}^{-3}$ (i.e. average for Dione), $d=5.6 \times 10^{5} \mathrm{~m}$, $g_{0}=0.23 \mathrm{~m} \mathrm{~s}^{-2}$. For the thermal diffusivity we assume $\kappa=3 \times 10^{-6} \mathrm{~m}^{2} \mathrm{~s}^{-1}$. The value of the sub-surface (subcrustal) viscosity $\eta_{\mathrm{s}}$ is critical. It should be higher than the viscosity in the bulk of the satellite interior. We assume $\eta_{\mathrm{s}}=10^{20} \mathrm{~kg} \mathrm{~m}^{-1} \mathrm{~s}^{-1}$. These values lead to $C \approx 5 \times$ $10^{-6}$, which could be treated as an upper limit of reasonable values of $C$. In most calculations we use two values of $C$; namely, $C=0$ and $C=4 \times 10^{-6}$ (see below). Of course, in any realistic case $C>0$ but for low sub-surface viscosity (i.e. lack of significant lithosphere) $C$ could be very low.

\section{Density Distribution for Steady-state Convec- tion}

We limit our considerations of density distribution to the most important quantities: the shift of the centre of mass, $\Delta r_{\mathrm{CM}}$, and the ratio of the moments of inertia, $I_{Z} / I_{X Y}$. Here $I_{Z}$ is the moment of inertia with respect to the axis of symmetry of the convective pattern and $I_{X Y}$ is the moment of inertia with respect to any axis in the 'equatorial' plane. Both $\Delta r_{\mathrm{CM}}$ and $I_{Z} / I_{X Y}$ could be determined by an observation of the gravitational field and the shape of the body. In the present model, $\Delta r_{\mathrm{CM}}$ and $I_{Z} / I_{X Y}$ are calculated by numerical integration. The density distribution as well as the surface deformation appearing in the integrals are obtained from the numerical model of convection.

The steady-state convection is determined by the value of $R a$. Our calculations are performed for $R a$ up to 300,000 . Higher values of $R a$ are improbable in the MIS. Note that the magnitude of the thermal as well as that the mechanical effects do not depend strongly on the nature of heating. Therefore, the results can be applied for both radiogenic and tidal heating. The assumption of a steady state is justified for both modes because: 

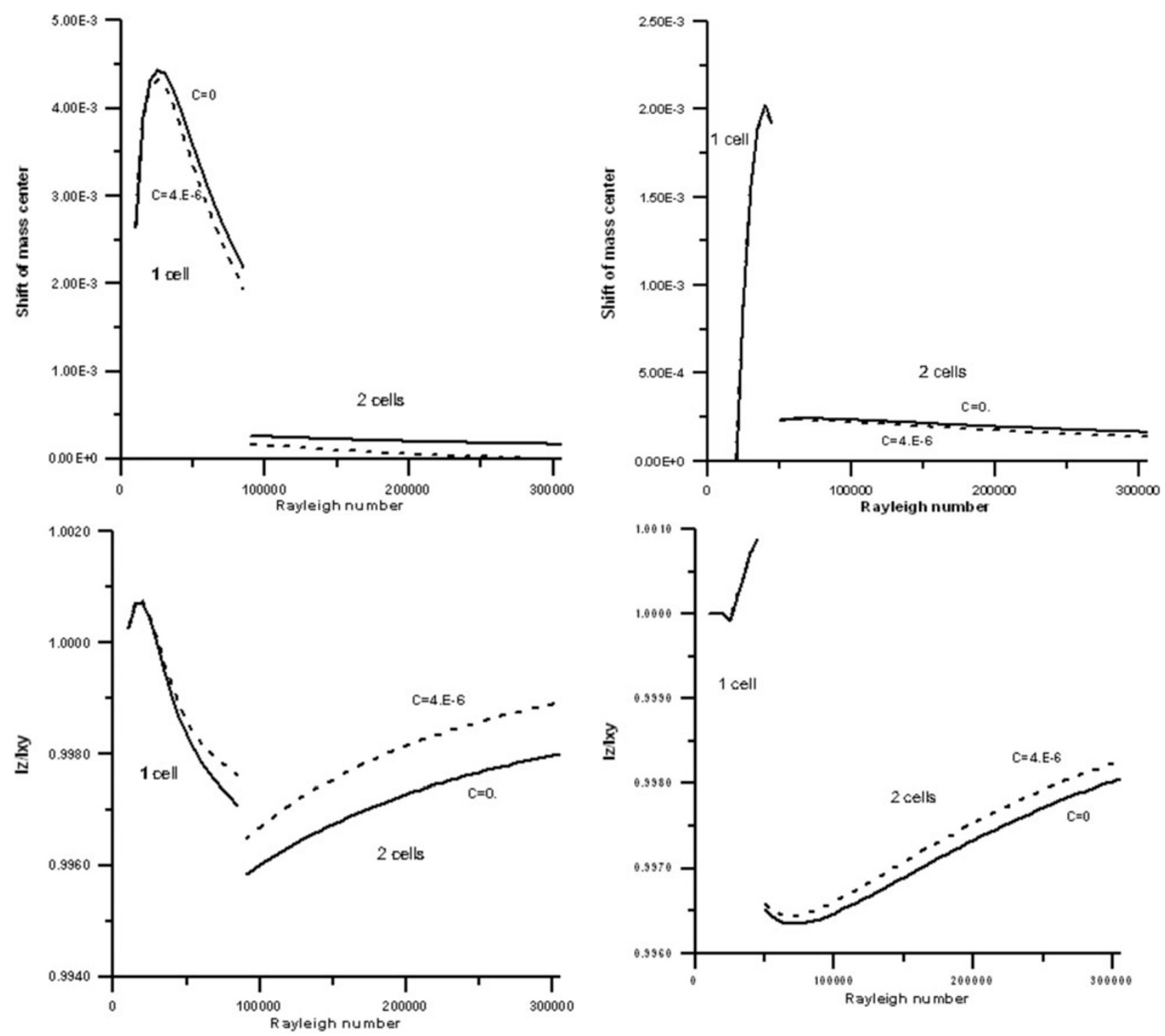

Fig. 3. The results for the free BC. The shift of the centre of mass $\Delta r_{\mathrm{CM}} / d$ (upper panel). The ratio of the moments of inertia $I_{Z} / I_{X Y}$ versus the Rayleigh number $R a$ (lower panel). The solid lines are for $C=0$; the dashed lines are for $C=4 \times 10^{-6}$. The discontinuity for $R a \approx 85000$ corresponds to the transformation of convection from the one-cell pattern to the two-cell pattern.

(1) The substantial tidal heating in the MIS is usually a result of forced eccentricity; this eccentricity could last billions of years.

(2) The radiogenic heating decreases very slowly in time.

The $\Delta r_{\mathrm{CM}}$ and $I_{Z} / I_{X Y}$ also depend on the number $C$. Figures 3 and 4 present $\Delta r_{\mathrm{CM}}$ (in the units of the satellite radius $d$ ) and the ratio $I_{Z} / I_{X Y}$ versus $R a$ for two different values of $C$ and for two different boundary conditions (free and rigid $\mathrm{BC}$ ). For low $R a$, the one-cell convective pattern is observed consisting of one region of cold (i.e. high density) downward current and one region of hot (i.e. low density) upward current (see Fig. 1). The density difference leads to a shift in the centre of mass towards denser regions. For increasing $R a$, up to 30,000 , the $\Delta r_{\mathrm{CM}}$ increases up to $0.0045 d$ (for the free BC; see Fig. 3). The deforma-

Fig. 4. The results for the rigid BC. The discontinuity for $R a \approx 45000$ corresponds to the transformation of convection from the one-cell pattern to the two-cell pattern. See Fig. 3 for details.

tion of the surface reduces this value only slightly (compare solid and dashed lines in the upper panel of Fig. 3). For the value of $R a$ increasing further, up to about 85,000 , the $\Delta r_{\mathrm{CM}}$ decreases. This decrease is a little surprising because the intensity of convection increases with $R a$. However, we have yet another effect: the thermal boundary layer (TBL) is thinning with increasing $R a$ (see, for example, Czechowski, 1993). For these changes in $\Delta r_{\mathrm{CM}}$ the effect of the TBL thinning eventually becomes more important than the increasing intensity of convection. For $R a \geq 90,000$ a twocell pattern appears and $\Delta r_{\mathrm{CM}}$ is dramatically lower. The low value of $\Delta r_{\mathrm{CM}}$ is a result of the approximate symmetry of the two-cell pattern with respect to the 'equatorial' plane (see Fig. 1). Note that these cells are not perfectly symmetric so $\Delta r_{\mathrm{CM}}>0$.

The ratio $I_{Z} / I_{X Y}$ is given in the lower panel of Fig. 3. For low $R a$, in the absence of convection, the ratio $I_{Z} / I_{X Y}=1$. For the one-cell pattern, the ratio $I_{Z} / I_{X Y}$ reaches a local maximum of 1.0007 at approximately $R a \approx 25,000$. Note 
that $\Delta r_{\mathrm{CM}}$ reaches its maximum for a slightly higher $R a$. Both quantities characterize different properties of density distribution; consequently, their values are different functions of $R a$. Then, for higher $R a$, up to about 85,000, this ratio decreases as a result of TBL thinning. For $R a=$ 90,000 (the lowest value of $R a$ found for the two-cell pattern), the ratio $I_{Z} / I_{X Y}$ is the smallest. It is a result of the concentration of low-density matter in the hot convection current below the 'equatorial' region. This region is located far from the $z$-axis (the axis of symmetry). This concentration of lighter matter significantly reduces $I_{Z}$ (compare the two-cell pattern in Fig. 1). The regions of higher density are close to the axis of symmetry, therefore their contribution to $I_{Z}$ is limited. However, these regions considerably increase $I_{X Y}$. For $R a>90,000$, the ratio $I_{Z} / I_{X Y}$ increases with $R a$, indicating again the effect of the thinning TBL.

Let us now focus on the case of the rigid BC (Fig. 4). The general behaviour of $\Delta r_{\mathrm{CM}}$ and $I_{Z} / I_{X Y}$ is similar to that with free $\mathrm{BC}$. The main difference is the value of $R a$ corresponding to the transition from one-cell pattern to twocell pattern. This transition is observed at $R a \approx 45000$. The effect of the TBL thinning is also observed-but mainly for the two-cell regime.

Our results indicate that the deviations of $\Delta r_{\mathrm{CM}}$ and $I_{Z} / I_{X Y}$ above $0.5 \%$ are improbable because higher $R a$ does not implicate higher deviations. Note that for reasonable values of the compensation number $C$, the deformation of the surface is not a deciding factor in determining $\Delta r_{\mathrm{CM}}$ and $I_{Z} / I_{X Y}$.

\section{The Evolution of Mass Distribution}

In order to investigate the evolution of convection, we need to specify the initial temperature for the system of Eqs. (7)-(9) and the time dependence of the heat sources $Q(t)$. We cannot simulate the evolution of convection driven by tidal heating because we do not know the time dependence of $q_{t}(r, \theta, \varphi)$. So, we assume here that $L=0$. Fortunately, the evolution of the radiogenic heating could be described by the following expression:

$$
\begin{aligned}
Q_{\mathrm{rad}}= & f_{M}\left[Q_{\mathrm{U} 235} \exp \left(-\lambda_{\mathrm{U} 235} t\right)+Q_{\mathrm{U} 238} \exp \left(-\lambda_{\mathrm{U} 238} t\right)\right. \\
& \left.+Q_{\mathrm{Th}} \exp \left(-\lambda_{\mathrm{Th}} t\right)+Q_{\mathrm{K} 40} \exp \left(-\lambda_{\mathrm{K} 40} t\right)\right],
\end{aligned}
$$

Where $f_{M}$ is the mass fraction of rock in the satellite material, $Q$ denotes concentrations of the radionuclide and $\lambda$ are their half-decay times. According to Prialnik et al. (1987) the values are: $Q_{\mathrm{U} 235}=1.14 \times 10^{-5} \mathrm{~J} \mathrm{~kg}^{-1} \mathrm{yr}^{-1}$, $\lambda_{\mathrm{U} 235}=9.71 \times 10^{-10} \mathrm{yr}, Q_{\mathrm{U} 238}=6.50 \times 10^{-5} \mathrm{~J} \mathrm{~kg}^{-1} \mathrm{yr}^{-1}$, $\lambda_{\mathrm{U} 238}=1.55 \times 10^{-10} \mathrm{yr}, Q_{\mathrm{Th}}=4.53 \times 10^{-5} \mathrm{~J} \mathrm{~kg}^{-1} \mathrm{yr}^{-1}$, $\lambda_{\mathrm{Th}}=4.99 \times 10^{-11} \mathrm{yr}, Q_{\mathrm{K} 40}=1.04 \times 10^{-3} \mathrm{~J} \mathrm{~kg}^{-1} \mathrm{yr}^{-1}$, $\lambda_{\mathrm{K} 40}=5.50 \times 10^{-10} \mathrm{yr}$. Note that the radionuclides are assumed to be distributed uniformly; therefore in the present case $q_{\text {av }}=q=Q_{\text {rad }} / c$.

The initial distribution of temperature is calculated assuming that the kinetic energy of the accreting matter is accumulated as heat inside a growing satellite. For an accreted satellite of uniform density (neglecting the heat conduction and the internal sources), the temperature distribution inside the growing satellite is given by (Schubert et al., 1986)

$$
T_{\text {ini }}(r)=T_{\text {neb }}+\chi 8 G \pi \rho r^{2} /\left(3 c_{\mathrm{p}}\right) .
$$

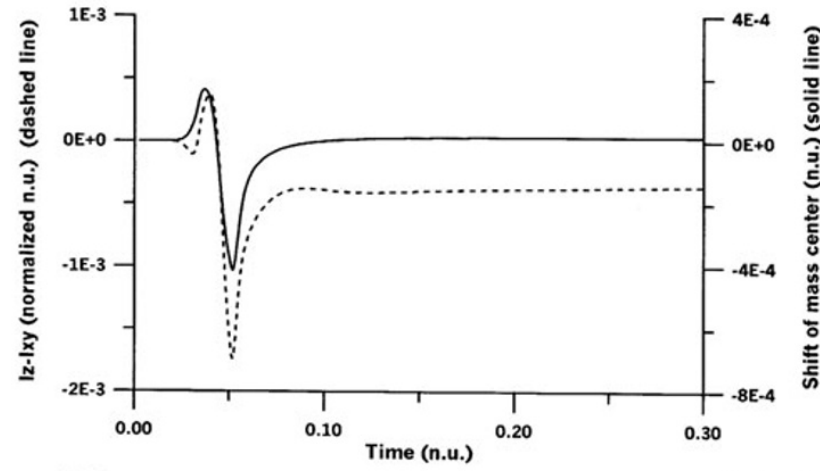

Fig. 5. The evolution of the distribution of mass for Dione. A hot origin and the free BC are assumed; $C=0.1 \times 10^{-6}, \eta=10^{18} \mathrm{~kg} \mathrm{~m}^{-1} \mathrm{~s}^{-1}$. Natural units are used for time, $\tau=2.27 \mathrm{Gyr}$, length, $d=560 \mathrm{~km}$, stream function, $\Psi=4 \times 10^{-4} \mathrm{~m}^{2} \mathrm{~s}^{-1}$ and temperature, $\Theta=801 \mathrm{~K}$. The upper panel shows $\Delta r_{\mathrm{CM}}$ (solid line) and the normalized difference of the moments of inertia $I_{Z}-I_{X Y}$ (dashed line). Note that the first convective cell shifts the centre of mass in one direction. The second cell with opposite sense of motion shifts the centre of mass in the opposite direction. The second cell prevails during a certain time interval. The lower panel shows the maximum values of the stream function (solid lines) and correspond to the clockwise and counter clockwise motion in the convective cells and the globally averaged temperature (dashed line).

Here, $T_{\text {neb }}$ is the temperature of the circum-planetary nebula. In the calculations, the coefficient of heat accumulation is assumed $\chi=0.5$.

The calculations were performed for Dione: $d=$ $560 \mathrm{~km}, \rho_{0}=1490 \mathrm{~kg} \mathrm{~m}^{-3}$ and $f_{M}=0.553$. Material parameters are assumed to be: $\alpha=10^{-4} \mathrm{~K}^{-1}, c=$ $1300 \mathrm{~J} \mathrm{~kg}^{-1} \mathrm{~K}^{-1}, \kappa=4.5 \times 10^{-6} \mathrm{~m}^{2} \mathrm{~s}^{-1}$. Here, we present two simulations. In the first simulation we assume hot origin (hot nebula) $T_{\text {neb }}=200 \mathrm{~K}$, and the viscosity of the satellite interior equals $\eta=10^{18} \mathrm{~kg} \mathrm{~m}^{-1} \mathrm{~s}^{-1}$. The second simulation starts with cold nebula, $T_{\text {neb }}=80 \mathrm{~K}$, and viscosity $\eta=4 \times 10^{18} \mathrm{~kg} \mathrm{~m}^{-1} \mathrm{~s}^{-1}$. Figure 5 presents the evolution (for the hot origin) of $I_{Z}-I_{X Y}, \Delta r_{\mathrm{CM}}$, the stream function and the globally averaged temperature of the satellite's interior. Initially, at $50 \mathrm{Myr}$ (i.e. $0.023 \tau$ ), the average temperature is high but convection cannot start because the temperature increases with central distance, according to Eq. (16). The internal heating, together with the surface cooling, eventually reverse the initial temperature gradient, and convection starts forming the one-cell pattern. It shifts the centre of the mass and leads to moderate changes in the moments of inertia. At $90 \mathrm{Myr}$ (i.e. $0.04 \tau$ ), we observe the formation of the second, very intensive convective cell 

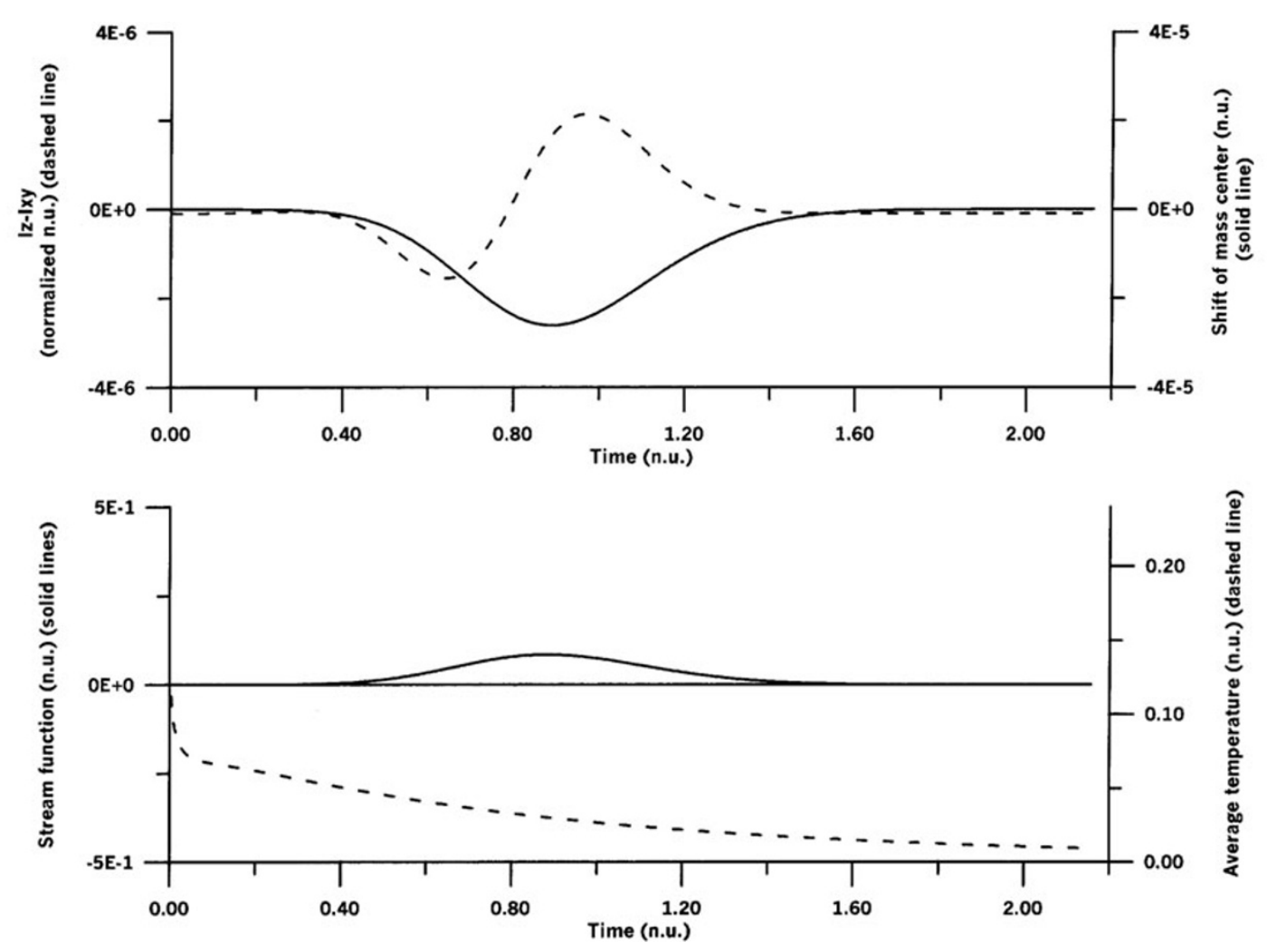

Fig. 6. The evolution of mass distribution for Dione. Cold origin and the free $\mathrm{BC}$ are assumed; $C=4 \times 10^{-6}, \eta=4 \times 10^{18} \mathrm{~kg} \mathrm{~m}^{-1} \mathrm{~s}^{-1}$. See Fig. 5 for details.

with opposite sense of motion. This cell predominates over the first one, leading to the opposite shift of the centre of mass. The $\Delta r_{\mathrm{CM}}$ then reaches the value of $4 \times 10^{-4}$ n.u. $(220$ $\mathrm{m})$. Moreover, the two-cell pattern leads to a considerable change in the moments of inertia. The period of intensive convection leads to intensive cooling of the interior (note the steep slope of the graph of the average temperature at time $0.05 \tau$ ). The period of very intensive convection ends at $0.07 \tau$, when absolute values of both graphs of stream functions drop to 2 n.u. At $160 \mathrm{Myr}(0.073 \tau)$, both cells show similar intensity. The two-cell pattern with a similar intensity of motion in both cells gives a very low value of $\Delta r_{\mathrm{CM}}$ (solid line) and a non-negligible difference between the moments of inertia $I_{Z}$ and $I_{X Y}$. Later, the evolution of convection just follows the evolution of the radiogenic heat sources $Q(t)$. Figure 6 presents the evolution for the cold origin, i.e. for $T_{\text {neb }}=80 \mathrm{~K}$. For this case, the convection starts later (at $0.4 \tau$, i.e. 900 Myr compared to $0.04 \tau$ illustrated on Fig. 5). It is the result of low initial temperature; the convection starts only when the average temperature increases sufficiently as a result of radiogenic heating. Note also that the convection shown in Fig. 6 is 200 times less intensive than that in Fig. 5 (compare graphs of stream functions). For such slow convection, only a one-cell pattern is possible, and the values of $I_{Z}-I_{X Y}$ and $\Delta r_{C M}$ are negligible in Fig. 6. The rescaling of the results for Dione for another satellite is possible, provided that the values of $\tau$ and $\Theta$ are the same. These conditions considerably limit the possibility of scaling. Therefore, we do not suggest that the evolution of the other MIS is the same as that of Dione.

\section{The Role of Impacts}

Impacts producing large craters lead to the mass redistribution in the MIS. A large impact ejects considerable volume and it forms a large crater. If the body's interior behaves like a fluid, then the isostasy considerably reduces the size of the cavity. The effects of isostasy are observed in most large craters on the MIS, suggesting that the fluid-like behaviour lasts for a long time after accretion. The best known example where the isostatic equilibrium is apparently not attained is Hershel, a major crater on Mimas. Mimas is the smallest of the MIS; there are no signs of tectonic activity, so the fact that its interior is rigid is not surprising.

Let us assume that a crater is a flat disk (penny-shaped) cavity. Simple calculations based on the definitions of the centre of mass and of the moments of inertia give:

$$
\begin{aligned}
& \left(\Delta r_{\mathrm{CM}}\right)_{\text {impact }} \approx(3 / 4) r_{\mathrm{cr}}^{2} h / d^{3}, \\
& \left(I_{Z} / I_{X Y}\right)_{\text {impact }} \approx I_{Z} / I_{X Y}\left[1+(15 / 8) r_{\mathrm{cr}}^{2} h / d^{3}\right],
\end{aligned}
$$

where $r_{\mathrm{cr}}$ is the radius of the crater and $h$ is its depth (the crater's centre is on the $z$-axis). Assuming $r_{\mathrm{cr}}=$ $d / 3$ and $h=d / 20$ (approximate data for the crater Herschel), we obtain $\left(\Delta r_{\mathrm{CM}}\right)_{\text {impact }} \approx 0.004 d$ (i.e. $800 \mathrm{~m}$ ) and $\left(I_{Z} / I_{X Y}\right)_{\text {impact }} \approx 1.01$. These values should be treated as the upper limits since a considerable volume of the material ejected from the crater forms the crater rim and the central peak. It should also be noted that Herschel is rather unique. Most large craters on the MIS have $r_{\mathrm{cr}} \leq 0.1 d$ and $h \leq$ $0.01 d$, so a single crater gives: $\left(\Delta r_{\mathrm{CM}}\right)_{\text {impact }} \leq 0.00007 d$ and $\left(I_{Z} / I_{X Y}\right)_{\text {impact }} \leq 1.0002$.

The impact cratering was the main cause of the non- 
spherical mass distribution in the Heavy Bombardment Epoch for small MIS. Note, however, that the total effect of impacts depends on the rheology of the interior of a given body. The rheology itself depends on temperature; for low temperature the viscosity is high and the interior behaves like a rigid body and even large impact craters could last on the geological time scale. This is the case of the Herschel crater on Mimas. The interior is expected to have a low viscosity at high temperature. The low viscosity results in two processes: an isostatic relaxation and convection. The isostatic relaxation of craters is an effective factor reducing the depth of the craters and, consequently, reducing the effect of impacts on the distribution of mass. On the other hand, the decreasing of viscosity boosts the Rayleigh numberthe effect that could lead to non-spherical mass redistribution induced by convection. Such a situation is possible for Enceladus. Concluding, a considerable role of impacts is expected for bodies where the role of convection is negligible, and vice-versa. Consequently, only one of the two factors - convection or impacts - could be significant.

\section{Conclusions}

(1) Convection could be responsible for the shift of the centre of the mass, $\Delta r_{\mathrm{CM}}$, up to $\sim 0.5 \%$ of the satellite radius and for the change of the ratio of the moments of inertia $I_{Z} / I_{X Y}$ up to $\sim 0.4 \%$.

(2) Convection driven by the radiogenic heating alone does not lead to high values of $\Delta r_{\mathrm{CM}}$ and $I_{Z} / I_{X Y}$. Convection driven by tidal heating could be much more effective due to stronger heating.

(3) For satellites close to the planet, the gravity of the planet could be responsible for the considerable amplification of $\Delta r_{\mathrm{CM}}$ and/or $I_{Z} / I_{X Y}$.

(4) For the smallest MIS, impact craters could give higher changes of $\Delta r_{\mathrm{CM}}$ and/or $I_{Z} / I_{X Y}$ than convection. For larger MIS, the isostatic relaxation reduces the effects of impact.

Acknowledgments. This work was supported by grant 0576/H03/2007/32 provided by Polish Ministry of Science and Education. We are grateful to the anonymous reviewers for their constructive remarks.

\section{References}

Czechowski, L., Simulation of a continent-continent collision using the mantle convection theory, Geophysica, 28, 41-51, 1992.
Czechowski, L., Theoretical approach to mantle convection, in Dynamics of the Earth's Evolution, edited by R. Teisseyre, L. Czechowski, and J. Leliwa-Kopystynski, pp. 161-271, Elsevier, Amsterdam, 1993.

Czechowski, L. and J. Leliwa-Kopystynski, Tidal heating and convection in the medium sized icy satellites, Celestial Mechanics Dynamical Astron., 87, 157-169, 2003.

Czechowski, L. and J. Leliwa-Kopystynski, Convection driven by tidal and radiogenic heating in medium size icy satellites, Planet. Space Sci., 53, 749-769, 2005.

Dermott, S. F. and P. C. Thomas, The shape and internal structure of Mimas, Icarus, 73, 25-65, 1988.

Ellsworth, K. and G. Schubert, Saturn icy satellites: thermal and structural models, Icarus, 54, 490-510, 1983.

Eluszkiewicz, J. and J. Leliwa-Kopystynski, Compression effects in rockice mixtures: an application to the study of satellites, Phys. Earth Planet. Inter., 55, 387-398, 1989.

Kossacki, K. J. and J. Leliwa-Kopystynski, Medium-size icy satellites: thermal and structural evolution during accretion, Planet. Space Sci., 41, 729-741, 1993

Leliwa-Kopystynski, J. and K. J. Kossacki, Evolution of porosity in small icy bodies, Planet. Space Sci., 48, 727-745, 2000.

Leliwa-Kopystynski, J., L. Makkonen, O. Erikoinen, and K. J. Kossacki, Kinetics of pressure induced effects in water ice/rock granular mixtures and application to the physics of icy satellites, Planet. Space Sci, $\mathbf{4 2}$, 545-555, 1994.

Leliwa-Kopystynski, J., M. Maruyama, and T. Nakajima, The waterammonia phase diagram up to $300 \mathrm{MPa}$ application to icy satellites, Icarus, 159, 518-528, 2002.

McKinnon, W. B., Geodynamics of Icy Satellites, in Solar System Ices, edited by B. Schmitt et al., pp. 525-550, Kluwer Academic Publishers, 1998

Peale, S. J., P. Cassen, and R. T. Reynolds, Melting of Io by tidal dissipation, Science, 203, 892-894, 1979.

Poirier, J. P., L. Boloh, and P. Chambon, Tidal dissipation in small viscoelastic ice moons: the case of Enceladus, Icarus, 55, 218-230, 1983.

Prialnik, D., A. Bar-Nun, and M. Podolak, Radiogenic heating of comets by Al26 and implications for their time of formation, Astrophys. J., $\mathbf{3 1 9}$, 993-1002, 1987.

Schubert, G., T. Spohn, and R. T. Reynolds, Thermal histories, compositions and internal structures of the moons of the Solar System, in Satellites, edited by J. A. Burns and M. S. Matthews, pp. 224-292, University Arizona Press, Tucson, 1986.

Schubert, G., D. L. Turcotte, and P. Olson, Mantle convection in the Earth and planets, Cambridge Univ. Press, Cambridge, UK, 2001.

Sotin, C., O. Grasset, and S. Beauchesne, Thermodynamical properties of high pressure ices. Implications for dynamic and internal structure of large icy satellites, in Solar System Ices, edited by B. Schmitt et al., pp. 79-96, Kluwer Academic Publishers, 1998.

Stacey, F. D., Physics of the Earth, Brookfield Press, Brisbane, Australia, 1992.

Zharkov, V. N., V. V. Leontiev, and A. V. Kozenko, Models, figures, and gravitational moments of the Galilean satellites of Jupiter and icy satellites of Saturn, Icarus, 62, 92-100, 1985.

L.Czechowski (e-mail: lczech@fuw.edu.pl) and J. Leliwa-Kopystynski (e-mail: jkopyst@mimuw.edu.pl) 